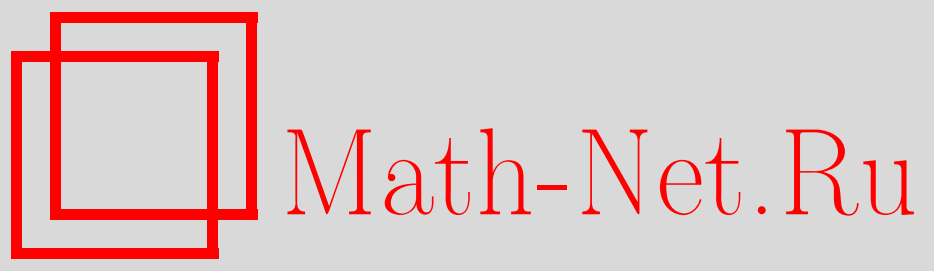

А. М. Грюндланд, Солитонные поверхности в подходе обобщенной симметрии, ТМФ, 2016, том 188, номер 3, 416-428

DOI: https://doi.org/10.4213/tmf9035

Использование Общероссийского математического портала Math-Net.Ru подразумевает, что вы прочитали и согласны с пользовательским соглашением http://www.mathnet.ru/rus/agreement

Параметры загрузки:

IP: 3.81 .55 .215

26 апреля 2023 г., 18:37:29

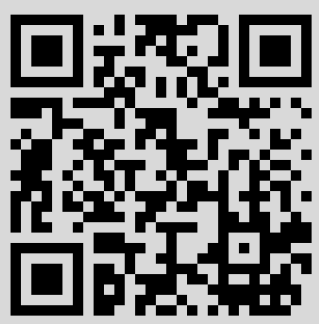




\title{
ФИЗИКА
}

Том 188, № 3

сентябрь, 2016

(C) 2016 г. $\quad$ А. М. Грюндланд ${ }^{* \dagger}$

\section{СОЛИТОННЫЕ ПОВЕРХНОСТИ В ПОДХОДЕ ОБОБЩЕННОЙ СИММЕТРИИ}

\begin{abstract}
Изучаются некоторые особенности обобщенных симметрий интегрируемых систем с целью получения формулы Фокаса-Гельфанда, описывающей погружение двумерных солитонных поверхностей в алгебры Ли. Показано, что если существует общая симметрия представления нулевой кривизны интегрируемого дифференциального уравнения в частных производных и его линейной спектральной задачи, то формула погружения Фокаса-Гельфанда является применимой в ее первоначальном виде. В общем случае показано, что если симметрия представления нулевой кривизны не является симметрией его линейной спектральной задачи, то функция погружения двумерной поверхности определяется расширенной формулой, включающей дополнительные члены в выражении для касательных векторов. Эти результаты проиллюстрированы примерами, включающими эллиптическое обыкновенное дифференциальное уравнение и уравнение $\mathbb{C} P^{N-1}$-сигма-модели.
\end{abstract}

Ключевые слова: интегрируемая система, солитонная поверхность, формула погружения, обобщенная симметрия.

DOI: $10.4213 / \operatorname{tmf} 9035$

\section{1. ПРЕДВАРИТЕЛЬНЫЕ СВЕДЕНИЯ О СОЛИТОННЫХ ПОВЕРХНОСТЯХ И ОБОБЩЕННЫХ СИММЕТРИЯХ ИНТЕГРИРУЕМЫХ СИСТЕМ}

Настоящая работа является продолжением исследований [1]-[9], посвященных теории солитонных поверхностей и обобщенных симметрий. Рассмотрим систему дифференциальных уравнений в частных производных, записанную в терминах двух независимых переменных $\xi_{1}, \xi_{2}$ и $m$ зависимых переменных $\theta^{k}\left(\xi_{1}, \xi_{2}\right)$ :

$$
\Omega[\theta]=0 .
$$

Работа была финансирована исследовательским грантом NSERC of Canada.

* Centre de Recherches Mathématiques Université de Montréal, Montréal, Canada. E-mail: grundlan@crm.umontreal.ca

${ }^{\dagger}$ Département de Mathématiques et d'Informatique Université du Québec à Trois-Rivières, Trois-Rivières, Canada 
Предположим, что система (1) может быть линеаризована с помощью матричной линейной спектральной задачи (ЛСЗ)

$$
\Lambda_{\alpha}([\theta], \lambda) \equiv D_{\alpha} \Phi([\theta], \lambda)-U_{\alpha}([\theta], \lambda) \Phi([\theta], \lambda)=0, \quad \lambda \in \mathbb{C}, \quad \alpha=1,2 ;
$$

условия совместности этих уравнений записываются как условие нулевой кривизны $(\mathrm{YHK})$

$$
\Omega[\theta]=D_{2} U_{1}-D_{1} U_{2}+\left[U_{1}, U_{2}\right]=0,
$$

которое считается не зависящим от $\lambda \in \mathbb{C}$. Мы полагаем, что волновая функция $\Phi([\theta], \lambda)$ и матрицы потенциала $U_{\alpha}([\theta], \lambda)$ могут быть определены на расширенном пространстве струй $\mathcal{N}=(N, \lambda)$. Здесь мы используем сокращенное обозначение пространства струй $N \ni[\theta]=\left(\xi_{1}, \xi_{2}, \theta^{k}, \theta_{J}^{k}\right)$,

$$
\frac{\partial^{n} \theta^{k}}{\partial \xi_{j_{1}} \ldots \partial \xi_{j_{n}}} \equiv \theta_{J}^{k}, \quad J=\left(j_{1}, \ldots, j_{n}\right), \quad j_{k} \in\{1,2\}, \quad|J|=n .
$$

Полные производные обозначаются следующим образом:

$$
D_{\alpha}=\frac{\partial}{\partial \xi_{\alpha}}+\theta_{J, \alpha}^{k} \frac{\partial}{\partial \theta_{J}^{k}}, \quad \alpha=1,2 .
$$

Здесь и ниже по повторяющимся индексам предполагается суммирование. Обобщенное векторное поле в эволюционном представлении и его расширение записываются в следующем виде:

$$
\omega_{R}=R^{k}[\theta] \frac{\partial}{\partial \theta^{k}}, \quad \operatorname{pr} \omega_{R}=R^{k}[\theta] \frac{\partial}{\partial \theta^{k}}+\left(D_{J} R^{k}[\theta]\right) \frac{\partial}{\partial \theta_{J}^{k}} .
$$

В работе [2] рассматривались двумерные поверхности общего вида, погруженные в полупростую алгебру Ли $\mathfrak{g}$ (которую можно отождествить с пространством $\mathbb{R}^{n}$ с соответствующим скалярным произведением), для которой уравнения Гаусса-Майнарди-Кодацци эквивалентны бесконечно малым деформациям $\Omega[\theta]=0$, и была найдена мгновенная бесконечно малая деформация ЛС3 (2) и УНК (3)

$$
\left|\begin{array}{c}
\widetilde{U}_{1} \\
\widetilde{U}_{2} \\
\widetilde{\Phi}
\end{array}\right|=\left|\begin{array}{c}
U_{1} \\
U_{2} \\
\Phi
\end{array}\right|+\epsilon\left(\begin{array}{c}
A_{1} \\
A_{2} \\
\Psi=\Phi F
\end{array}\right), \quad 0<\epsilon \ll 1,
$$

где $\widetilde{U}_{1}, \widetilde{U}_{2}, A_{1}, A_{2}, F \in \mathfrak{g}$ и $\widetilde{\Phi}=\Phi(\mathbb{I}+\epsilon F) \in G$. Матричные функции $\left(\widetilde{U}_{1}, \widetilde{U}_{2}, \widetilde{\Phi}\right)^{\mathrm{T}}$ должны иметь ту же структуру сингулярности в точке $\lambda$, что и $\left(U_{1}, U_{2}, \Phi\right)^{\mathrm{T}}$. Подставляя (7) в УНК (3) и в ЛС3 (2) и сохраняя члены первого порядка по $\epsilon$, получаем бесконечно малую деформацию УНК (3). Это приводит к следующему требованию: матричные функции $A_{1}$ и $A_{2}$ должны удовлетворять выражению

$$
D_{2} A_{1}-D_{1} A_{2}+\left[A_{1}, U_{2}\right]+\left[U_{1}, A_{2}\right]=0 .
$$

Из бесконечно малой деформации УНК (2) следует, что функция $\Psi$ удовлетворяет уравнению

$$
D_{\alpha} \Psi=U_{\alpha} \Psi+A_{\alpha} \Phi, \quad \alpha=1,2 .
$$


Требование (8) совпадает с условием совместности системы (9). Тогда с учетом соотношений (7)-(9) условие существования $\mathfrak{g}$-значной функции погружения двумерной поверхности в $\mathfrak{g}$ вида

$$
F=\left.\left(\Phi^{-1} \frac{d \tilde{\Phi}}{d \epsilon}\right)\right|_{\epsilon=0} \in \mathfrak{g}
$$

может быть выражено в терминах матриц $A_{1}$ и $A_{2}$, которые удовлетворяют (8) и (9). Эти результаты приводят к следующим теоремам.

Теорема 1 (Фокас, Гельфанд [2]). Предположим, что существуют две $\mathfrak{g}$-значные матричные функиии $U_{1}, U_{2}$ и $G$-значная функция $\Phi$, выбранная так, чтобъ удовлетворять УНК (3) и связанной с ним ЛСЗ (2). Также предположим, что g-значные матрицы $A_{1}$ и $A_{2}$ являются линейно независимыми и удовлетворяют бесконечно малой деформации УНК (8). Тогда существует двумерная поверхность с такой $\mathfrak{g}$-значной функцией погружения $F([\theta], \lambda)$, что касательные векторы даются выражением

$$
D_{\alpha} F([\theta], \lambda)=\Phi^{-1} A_{\alpha}([\theta], \lambda) \Phi, \quad \alpha=1,2 .
$$

Первая и вторая фундаментальные формы поверхности можно выразить только в терминах $U_{\alpha}, A_{\alpha}$.

Под интегрируемъми поверхностями мы понимаем поверхности, связанные с интегрируемыми уравнениями Гаусса-Майнарди-Кодацци.

Следующая теорема представляет собой основной результат по погружению поверхностей.

ТЕОРЕМа 2 (Фокас и др. [3]). Пусть множество скалярных функиий $\left\{\theta^{j}\right\}$ удовлетворяет интегрируемым нелинейным дифберенииальным уравнениям $\Omega[\theta]=0$ u пусть $G$-значная функиия $\Phi([\theta], \lambda)$ удовлетворяет ЛСЗ (2), где $\mathfrak{g}$-значнье функици $U_{\alpha}([\theta], \lambda)$ удовлетворяют УНК (3). Определим линейно независимые $\mathfrak{g}$-значные матричные функции $A_{\alpha}([\theta], \lambda)$ :

$$
A_{\alpha}([\theta], \lambda)=\beta(\lambda) D_{\lambda} U_{\alpha}+\left(D_{\alpha} S+\left[S, U_{\alpha}\right]\right)+\frac{D U_{\alpha}}{D \theta^{j}} R^{j} \in \mathfrak{g}, \quad \alpha=1,2,
$$

где $\beta(\lambda)$ - произвольная скалярная функция от $\lambda, S([\theta], \lambda)$ - произвольная $\mathfrak{g}$-значная матричная функиия, определенная на пространстве струй $(N, \lambda), R^{k}[\theta]$ - обобщенные симметрии нелинейных дифференциальных уравнений в частных производнъх (1), $\left(D U_{\alpha} / D \theta^{j}\right) R^{j}$ - производные Фреше от $U_{\alpha}$ в направлениях $R^{k}$. Тогда существует двумерная поверхность с функцией погружения $F([\theta], \lambda)$ в алгебру Ли $\mathfrak{g}$, которая определяется формулой

$$
F([\theta], \lambda)=\Phi^{-1}\left(\beta(\lambda) D_{\lambda} \Phi+S \Phi+\frac{D \Phi}{D \theta^{j}} R^{j}\right) \in \mathfrak{g}
$$

(с точностъю до аддитивной $\mathfrak{g}$-значной константы).

Интегральная форма (13) определяет отображение $F:(N, \lambda) \rightarrow \mathfrak{g} ;$ мы будем ссылаться на нее как на формулу погружения Фокаса-Гельфанда. Три слагаемых 
в скобках в формуле (13) соответствуют сдвигу спектрального параметра $\lambda$ (формула Сыма-Тафеля для погружения [4]), калибровочной симметрии ЛС3 (2) [5], [6] и обобщенным симметриям интегрируемых дифференциальных уравнений (1) [2].

Ниже мы будем рассматривать случай $\beta=S=0$. Формула погружения, связанная с обобщенными симметриями интегрируемых дифференциальных уравнений в частных производных (1), дается выражением

$$
F([\theta], \lambda)=\Phi^{-1} \frac{D \Phi}{D \theta^{j}} R^{j} \in \mathfrak{g},
$$

где касательные векторы имеют вид

$$
D_{\alpha} F([\theta], \lambda)=\Phi^{-1} A_{\alpha}([\theta], \lambda) \Phi, \quad A_{\alpha}([\theta], \lambda)=\frac{D U_{\alpha}}{D \theta^{j}} R^{j} \in \mathfrak{g} .
$$

\section{2. ОБОБЩЕННЫЕ СИММЕТРИИ И ФОРМУЛА ПОГРУЖЕНИЯ ФОКАСА-ГЕЛЬФАНДА}

Покажем, когда справедлива формула погружения Фокаса-Гельфанда в ее первоначальном виде. Векторное поле $\omega_{R}$, записанное в эволюционном виде и определенное на пространстве струй $N$, является симметрией УНК (3) тогда и только тогда, когда

$$
\begin{aligned}
\operatorname{pr} \omega_{R}\left(D_{2} U_{1}-D_{1} U_{2}+\left[U_{1}, U_{2}\right]\right)= & D_{2}\left(\operatorname{pr} \omega_{R} U_{1}\right)-D_{1}\left(\operatorname{pr} \omega_{R} U_{2}\right)+ \\
& +\left[\operatorname{pr} \omega_{R} U_{1}, U_{2}\right]+\left[U_{1}, \operatorname{pr} \omega_{R} U_{2}\right]=0,
\end{aligned}
$$

если $\Omega[\theta]=D_{2} U_{1}-D_{1} U_{2}+\left[U_{1}, U_{2}\right]=0$. Выражение (16) эквивалентно бесконечно малой деформации $\mathrm{УHK} \mathrm{(8)} \mathrm{с}$

$$
A_{\alpha}=\operatorname{pr} \omega_{R} U_{\alpha}, \quad \alpha=1,2,
$$

так как имеют место уравнения

$$
\left[D_{\alpha}, \operatorname{pr} \omega_{R}\right]=0, \quad \alpha=1,2 .
$$

Производная Фреше от $\Phi$ по $\theta^{k}$ в направлении $R^{k}$ может быть выражена в терминах расширения векторного поля $\omega_{R}[7]$ :

$$
F=\Phi^{-1} \frac{D \Phi}{D \theta^{k}} R^{k}=\Phi^{-1}\left(\operatorname{pr} \omega_{R} \Phi\right) .
$$

Дифференцируя (19) и используя ЛСЗ (2), мы получаем

$$
D_{\alpha} F=D_{\alpha}\left(\Phi^{-1} \frac{D \Phi}{D \theta^{k}} R^{k}\right)=\Phi^{-1}\left[-U_{\alpha} \frac{D \Phi}{D \theta^{k}} R^{k}+D_{\alpha}\left(\frac{D \Phi}{D \theta^{k}} R^{k}\right)\right] .
$$

Используя соотношения (18) и (19), мы можем записать второй член в правой части (20) как

$$
D_{\alpha}\left(\frac{D \Phi}{D \theta^{k}} R^{k}\right)=D_{\alpha}\left(\operatorname{pr} \omega_{R} \Phi\right)=\operatorname{pr} \omega_{R}\left(D_{\alpha} \Phi\right) .
$$

Применяя тождество

$$
\operatorname{pr} \omega_{R}\left(D_{\alpha} \Phi\right)=\operatorname{pr} \omega_{R}\left(U_{\alpha} \Phi\right)+\operatorname{pr} \omega_{R}\left(D_{\alpha} \Phi-U_{\alpha} \Phi\right)
$$


мы обнаруживаем, что второй член в (22) не обязательно обращается в нуль. Этот член обращается в нуль тогда и только тогда, когда векторное поле $\omega_{R}$ также является симметрией ЛСЗ $\Lambda([\theta], \lambda)=0$, т. е.

$$
\operatorname{pr} \omega_{R}\left(D_{\alpha} \Phi-U_{\alpha} \Phi\right)=0, \quad \text { если } \quad D_{\alpha} \Phi-U_{\alpha} \Phi=0 .
$$

Если мы считаем, что равенство (23) имеет место, то из (22) мы получаем

$$
\begin{aligned}
\operatorname{pr} \omega_{R}\left(D_{\alpha} \Phi\right)=\operatorname{pr} \omega_{R}\left(U_{\alpha} \Phi\right) & =\left(\operatorname{pr} \omega_{R} U_{\alpha}\right) \Phi+U_{\alpha}\left(\operatorname{pr} \omega_{R} \Phi\right)= \\
& =\left(\frac{D U_{\alpha}}{D \theta^{k}} R^{k}\right) \Phi+U_{\alpha}\left(\frac{D \Phi}{D \theta^{k}} R^{k}\right) .
\end{aligned}
$$

Подставляя (24) в (20) и используя уравнения (17), мы получаем касательные векторы $D_{\alpha} F$ в виде, заданном в теореме 1 :

$$
\begin{aligned}
D_{\alpha} F & =\Phi^{-1}\left[-U_{\alpha}\left(\frac{D \Phi}{D \theta^{k}} R^{k}\right)+\left(\frac{D U_{\alpha}}{D \theta^{k}} R^{k}\right)+U_{\alpha}\left(\frac{D \Phi}{D \theta^{k}} R^{k}\right) \Phi\right]= \\
& =\Phi^{-1}\left(\frac{D U_{\alpha}}{D \theta^{k}} R^{k}\right) \Phi=\Phi^{-1}\left(\operatorname{pr} \omega_{R} U_{\alpha}\right) \Phi=\Phi^{-1} A_{\alpha}([\theta], \lambda) \Phi
\end{aligned}
$$

Таким образом, в предположении, что $\omega_{R}$ является общей симметрией УНК (3) и ЛС3 (2) (см. приложение), существует двумерная поверхность с g-значной функцией погружения, задаваемой выражением [8]

$$
F=\Phi^{-1}\left(\operatorname{pr} \omega_{R} \Phi\right)
$$

Следовательно, формула погружения Фокаса-Гельфанда может быть применена в своем первоначальном виде.

Рассмотрим векторное поле $\eta_{Q}$ в эволюционном виде, определенное на пространстве струй $M=[(\Phi, U)]$ :

$$
\eta_{Q}=Q^{j}[(\Phi, U)] \frac{\partial}{\partial \Phi^{j}}+\widehat{Q}_{\alpha}^{j}[(\Phi, U)] \frac{\partial}{\partial U_{\alpha}^{j}},
$$

где $\Phi=e^{\Phi^{j}} e_{j} \in G, U_{\alpha}=U_{\alpha}^{j} e_{j} \in \mathfrak{g}, \widehat{Q}_{\alpha}[(\Phi, U)]=\widehat{Q}_{\alpha}^{j}[(\Phi, U)] e_{j} \in \mathfrak{g}$. Предположим, что $\eta_{Q}$ является обобщенной симметрией ЛСЗ (2):

$$
\operatorname{pr} \eta_{Q}\left(D_{\alpha} \Phi-U_{\alpha} \Phi\right)=0, \quad \text { если } \quad D_{\alpha} \Phi-U_{\alpha} \Phi=0, \quad \alpha=1,2 .
$$

Векторные поля $\omega_{R}$ и $\eta_{Q}$ являются $\tau$-связанными в том смысле, что для любой параметризации $\Phi([\theta], \lambda)$ и $U_{\alpha}([\theta], \lambda)$ в терминах пространства струй $(N, \lambda)$ мы получаем отображение $\tau: N \rightarrow M$, которое считается диффеоморфизмом в $M$. Эта параметризация допустима тогда и только тогда, когда

$$
\operatorname{pr} d \tau\left(\omega_{R}\right)\left(D_{\alpha} \Phi-U_{\alpha} \Phi\right)=0, \quad \text { если } \quad \Omega[\theta]=0 .
$$

Требование, что поле $\omega_{R}$ является $\tau$-связанным с $\eta_{Q}$, означает, что продолжение $\omega_{R}$ должно быть векторным полем $\eta_{Q}$ на отображении $\tau$. Тогда $d \tau\left(\omega_{R}\right)$ является хорошо определенным векторным полем на $M$,

$$
d \tau\left(\omega_{R}\right)=\left.\eta_{Q}\right|_{\tau}, \quad[\theta] \in N .
$$


В координатном представлении это выражение принимает вид

$$
d \tau\left(\omega_{R}\right)=R^{k}[\theta]\left(\frac{\partial \Phi^{j}}{\partial \theta^{k}} \frac{\partial}{\partial \Phi^{j}}+\frac{\partial U_{\alpha}^{j}}{\partial \theta^{k}} \frac{\partial}{\partial U_{\alpha}^{j}}\right) .
$$

Таким образом, требование, что векторные поля $\omega_{R}$ и $\eta_{Q}$ являются $\tau$-связанными (30), эквивалентно следующим соотношениям:

$$
\omega_{R}=\left.R^{k}[\theta] \frac{\partial}{\partial \theta^{k}} \quad \eta_{Q}\right|_{\tau}=R^{k}[\theta] \frac{\partial \Phi^{j}}{\partial \theta^{k}} \frac{\partial}{\partial \Phi^{j}}+R^{k}[\theta] \frac{\partial U_{\alpha}^{j}}{\partial \theta^{k}} \frac{\partial}{\partial U_{\alpha}^{j}} .
$$

ПрЕДЛОЖЕНИЕ 1 [1]. Пусть существуют два векторнъх поля $\eta_{Q} u \omega_{R}$ такие, что $\eta_{Q}$, определенное на пространстве струй $M=[(\Phi, U)]$, является обобщенной симметрией ЛСЗ $\Lambda([\theta], \lambda)=0$, в то время как $\omega_{R}$, определенное на пространстве струй $N=[\theta]$, является обобщенной симметрией интегрируемого дифберенциального уравнения в частных производных $\Omega[\theta]=0$. Если векторные поля $\omega_{R}$ и $\eta_{Q}$ являются $\tau$-связанными, то векторное поле $\omega_{R}$ является общей симметрией как для уравнения $\Omega[\theta]=0$, так и для уравнения $\Lambda([\theta], \lambda)=0$.

Доказательство следует из цепного правила и того факта, что

$$
\sigma_{R} \equiv d \tau\left(\omega_{R}\right)=\left.\eta_{Q}\right|_{\tau}, \quad[\theta] \in N
$$

А именно,

$$
\begin{aligned}
\operatorname{pr} d \tau\left(\omega_{R}\right)\left(D_{\alpha} \Phi-U_{\alpha} \Phi\right) & =\operatorname{pr} \sigma_{R}\left(D_{\alpha} \Phi-U_{\alpha} \Phi\right) \operatorname{pr} \omega_{R}\left(D_{\alpha} \Phi-U_{\alpha} \Phi\right)= \\
& =\left.\operatorname{pr} \eta_{Q}\left(D_{\alpha} \Phi-U_{\alpha} \Phi\right)\right|_{\tau}=0, \quad \text { если } \quad \Omega[\theta]=0 .
\end{aligned}
$$

Таким образом, если векторные поля $\omega_{R}$ и $\eta_{Q}$ являются $\tau$-связанными, то существует симметрия уравнения $\Omega[\theta]=0$, которая связана как группа с обобщенной симметрией ЛС3 $\Lambda([\theta], \lambda)=0$ и таким образом индуцирует бесконечно малую деформацию функции $\Phi$. Тогда интегральная форма поверхности дается выражением (26), что согласуется с касательными векторами

$$
D_{\alpha} F([\theta], \lambda)=\Phi^{-1}\left(\operatorname{pr} \omega_{R} U_{\alpha}\right) \Phi, \quad \alpha=1,2 .
$$

Рассмотрим случай, когда векторное поле $\omega_{R}$ является симметрией ЛСЗ $\Omega[\theta]=0$, но не симметрией ЛС3 $\Lambda([\theta], \lambda)=0$ (т. е. действие $\operatorname{pr} d \tau\left(\omega_{R}\right)$ на ЛС3 не равно нулю для всех решений $\Phi$ линейной спектральной задачи). Если касательные векторы (35) являются линейно независимыми, то существует $\mathfrak{g}$-значная функция погружения $F$ для двумерной поверхности. Однако интегральная форма поверхности не является формой, задаваемой выражениями (26), (35). Следовательно, погружение $F$ не является погружением Фокаса-Гельфанда.

В общем случае, когда векторное поле $\omega_{R}$ является симметрией ЛС3 $\Omega[\theta]=0$, но не обязательно симметрией УНК $\Lambda([\theta], \lambda)=0$, мы имеем следующий результат.

ПрЕДЛОЖЕНИЕ 2. Если векторное поле $\omega_{R}$ является обобщенной симметрией ЛСЗ $\Omega[\theta]=0$, а $\mathfrak{g}$-значные матрицы

$$
A_{\alpha}=\operatorname{pr} \omega_{R} U_{\alpha}+\left(\operatorname{pr} \omega_{R}\left(D_{\alpha} \Phi-U_{\alpha} \Phi\right)\right) \Phi^{-1}, \quad \alpha=1,2,
$$


являются линейно независимыми, то функиия погружения $F$ двумерной поверхности определяется формулой (с точностъю до аддитивной $\mathfrak{g}$-значной константы)

$$
F([\theta], \lambda)=\Phi^{-1}\left(\operatorname{pr} \omega_{R} \Phi\right) \in \mathfrak{g},
$$

согласующейся с условием тангенциальности векторов

$$
D_{\alpha} F=\Phi^{-1}\left\{\left(\operatorname{pr} \omega_{R} U_{\alpha}\right) \Phi+\operatorname{pr} \omega_{R}\left(D_{\alpha} \Phi-U_{\alpha} \Phi\right)\right\}=\Phi^{-1}\left(D_{\alpha}-U_{\alpha}\right)\left(\operatorname{pr} \omega_{R} \Phi\right) .
$$

ДокАзАтЕльство. Уравнение (8) есть в точности уравнение совместности для условий (38) с матрицами $A_{\alpha}$, заданными в (36), и, таким образом, функция погружения $F$ существует и определяется выражением (37) с точностью до аддитивной $\mathfrak{g}$-значной константы.

\section{3. ПРИМЕНЕНИЕ МЕТОДА}

Для применения метода построения солитонных поверхностей требуется наличие трех составляющих явного представления функции погружения $F$, а именно нам потребуются:

1) представление нулевой кривизны дифференциального уравнения $\Omega[\theta]=0$;

2) обобщенная симметрия дифференциального уравнения $\Omega[\theta]=0$;

3) решение $\Phi$ ЛСЗ $\Lambda([\theta], \lambda)=0$.

Заметим, что первое условие должно выполняться всегда. Однако даже без двух других условий можно проводить некоторый анализ функции погружения $F$ (заданной выражением (13))в алгебру Ли $\mathfrak{g}$.

1. Без решения $\Phi$ ЛС3 (2) геометрия поверхности $F$ может быть проанализирована с помощью невырожденной формы Киллинга на алгебре Ли $\mathfrak{g}$ (в предположении, что $\mathfrak{g}$ является прямой суммой простых алгебр Ли). Функция погружения $F$ интерпретируется как псевдориманово многообразие. Касательные векторы и нормальный вектор к поверхности задаются как

$$
D_{\alpha} F=\Phi^{-1} A_{\alpha} \Phi \in \mathfrak{g}, \quad N=\frac{\Phi^{-1}\left[A_{1}, A_{2}\right] \Phi}{\left(\left[A_{1}, A_{2}\right]^{2} / 2\right)^{1 / 2}} \in \mathfrak{g}, \quad A_{\alpha}=\operatorname{pr} \omega_{R} U_{\alpha}
$$

где $\omega_{R}$ является общей симметрией УНК $\Omega[\theta]=0$ и ЛСЗ $\Lambda([\theta], \lambda)=0$.

Первая и вторая основные формы задаются с помощью выражений

$$
\begin{aligned}
& \mathrm{I}= \frac{\epsilon}{2}\left(\operatorname{tr}\left(A_{1}^{2}\right) d \xi_{1}^{2}+2 \operatorname{tr}\left(A_{1} A_{2}\right) d \xi_{1} d \xi_{2}+\operatorname{tr}\left(A_{2}^{2}\right) d \xi_{2}^{2}\right) \\
& \mathrm{II}=\frac{\epsilon}{2}\left\{\operatorname{tr}\left[\left(D_{1} A_{1}+\left[A_{1}, U_{1}\right]\right) N\right] d \xi_{1}^{2}+2 \operatorname{tr}\left[\left(D_{2} A_{1}+\left[A_{1}, U_{2}\right]\right) N\right] d \xi_{1} d \xi_{2}+\right. \\
&\left.\quad+\operatorname{tr}\left[\left(D_{2} A_{2}+\left[A_{2}, U_{2}\right]\right) N\right] d \xi_{2}^{2}\right\}
\end{aligned}
$$

где $\epsilon= \pm 1$.

2. Если мы имеем решение $\Phi$ ЛС3, но не обобщенную симметрию $\omega_{R}$ дифференциального уравнения в частных производных, то мы всегда можем рассматривать двумерную поверхность

$$
F=\Phi^{-1}\left(\beta(\lambda) D_{\lambda} \Phi+S \Phi\right), \quad S \in \mathfrak{g}, \quad \Phi \in G,
$$


определенную с помощью калибровочного преобразования и сдвига по $\lambda$ в УНК, которой отвечают касательные векторы

$$
D_{\alpha} F=\Phi^{-1}\left(\beta(\lambda) D_{\lambda} U_{\alpha}+D_{\alpha} S+\left[S, U_{\alpha}\right]\right) \Phi
$$

\section{4. ЭЛЛИПТИЧЕСКИЕ УРАВНЕНИЯ И СОЛИТОННЫЕ ПОВЕРХНОСТИ}

Рассмотрим следующее автономное обыкновенное дифференциальное уравнение второго порядка для функции $\theta$ относительно независимой переменной $x$ :

$$
\Omega[\theta]=\theta_{x x}-\frac{1}{2} f^{\prime}(\theta)=0, \quad f^{\prime}(\theta)=\frac{d}{d \theta} f(\theta),
$$

для некоторой функции $f^{\prime}(\theta)$. Уравнение (44) допускает первый интеграл

$$
\theta_{x}-\epsilon[f(\theta)]^{1 / 2}=0, \quad \epsilon= \pm 1
$$

и решения (45) удовлетворяют уравнению

$$
\epsilon \int_{\theta_{0}}^{\theta}[f(s)]^{-1 / 2} d s=x-x_{0}
$$

Если $[f(\theta)]^{-1 / 2}=w\left(\theta,\left[P_{n}(\theta)\right]^{1 / 2}\right)$, где $w$ - рациональная функция своего аргумента, а $P_{n}(\theta)$ - полином третьей или четвертой степени, а функция $\theta$, которая удовлетворяет (44), является обратная к эллиптической функции.

Обыкновенное дифференциальное уравнение (44) допускает матричную ЛСЗ при $\Phi \in S L(2, \mathbb{R}):$

$$
\Lambda([\theta], y, \lambda)=\left\{\begin{array}{l}
D_{x} \Phi([\theta], y, \lambda)=U_{1}([\theta], \lambda) \Phi([\theta], y, \lambda), \\
D_{y} \Phi([\theta], y, \lambda)=U_{2}([\theta], \lambda) \Phi([\theta], y, \lambda),
\end{array} \quad \lambda \in \mathbb{R}\right.
$$

где

$$
D_{x}=\frac{\partial}{\partial x}+\theta_{x} \frac{\partial}{\partial \theta}+\theta_{x x} \frac{\partial}{\partial \theta_{x}}, \quad D_{y}=\frac{\partial}{\partial y}, \quad\left[D_{x}, D_{y}\right]=0,
$$

так как $\theta_{y}=0$. Заметим, что мы ввели дополнительную переменную $y$ в ЛС3 (47) так, чтобы решение $\Phi$ зависело от $y$, а функции $U_{\alpha}$ от $y$ не зависели. То есть $D_{y} U_{\alpha}([\theta], \lambda)=0, \alpha=1,2$. Следовательно, УНК $\Omega[\theta]=0$ принимает вид

$$
\Omega[\theta]=D_{y} U_{1}-D_{x} U_{2}+\left[U_{1}, U_{2}\right]=D_{x} U_{2}+\left[U_{2}, U_{1}\right]=0 .
$$

Матричные функции $U_{\alpha} \in \mathfrak{s l}(2, \mathbb{R})$, удовлетворяющие уравнению (49), имеют вид [9]

$$
\begin{aligned}
& U_{1}([\theta], \lambda)=\frac{1}{2}\left(\begin{array}{cc}
0 & \frac{f^{\prime}(\theta)}{\theta+\lambda}-\frac{f(\theta)-f(-\lambda)}{(\theta+\lambda)^{2}} \\
1 & 0
\end{array}\right), \\
& U_{2}([\theta], \lambda)=\left(\begin{array}{cc}
\theta_{x} & -\frac{f(\theta)-f(-\lambda)}{\theta+\lambda} \\
\theta+\lambda & -\theta_{x}
\end{array}\right),
\end{aligned}
$$


где $f(\cdot)$ - полином. Частное решение $\Phi$ ЛС3 (47) с матрицами, задаваемыми выражениями (50), записывается как [1]

$$
\Phi([\theta], y, \lambda)=\left(\begin{array}{cc}
2 D_{x} \psi_{+} & 2 D_{x} \psi_{-} \\
\psi_{+} & \psi_{-}
\end{array}\right) \in \operatorname{sl}(2, \mathbb{R})
$$

где функции

$$
\psi_{ \pm}=\frac{(\theta+\lambda)^{1 / 2}}{(2 a)^{1 / 2}} \exp \left[ \pm a\left(y+\int_{\theta_{0}}^{\theta} \frac{d s}{2 \epsilon(s+\lambda)[f(s)]^{1 / 2}}\right)\right], \quad a=[f(-\lambda)]^{1 / 2}, \quad \epsilon= \pm 1,
$$

удовлетворяют следующей системе дифференциальных уравнений:

$$
\begin{aligned}
D_{x}^{2} \psi_{ \pm}-\frac{1}{4}\left(\frac{f^{\prime}}{\theta-\lambda}-\frac{f-a^{2}}{(\theta+\lambda)^{2}}\right) \psi_{ \pm} & =0 \\
\left(D_{x} \psi_{+}\right)\left(D_{x} \psi_{-}\right)-\frac{f-a^{2}}{8 a(\theta+\lambda)} & =0 .
\end{aligned}
$$

Уравнения (52) могут быть записаны в терминах функции $\theta$ и ее производных по $x$ :

$$
\begin{gathered}
\theta_{x x}-\frac{\theta_{x}^{2}}{2(\theta+\lambda)}-\frac{f^{\prime}}{2}+\frac{f-a^{2}}{2(\theta+\lambda)}+a\left(\frac{\theta_{x x}}{\epsilon \sqrt{f}}-\frac{\theta_{x}^{2} f^{\prime}}{2 \epsilon f^{3 / 2}}\right)+\frac{a^{2} \theta_{x}^{2}}{2 f(\theta+\lambda)}=0, \\
\theta_{x}^{2}-a^{2} \frac{\theta_{x}^{2}}{f}-f+a^{2}=0 .
\end{gathered}
$$

Уравнения (53) должны быть справедливы при всех значениях а. Отсюда следует, что

$$
\theta_{x x}-\frac{1}{2} f^{\prime}(\theta)=0, \quad \theta_{x}^{2}-f(\theta)=0 .
$$

В свою очередь, из уравнений (54) следует, что симметрия УНК $\Omega[\theta]=0$ является общей симметрией ЛС3 (47) тогда и только тогда, когда она является обобщенной симметрией выражения (54). Обобщенные симметрии уравнения (44) определяются бесконечно малой деформацией УНК (49), задаваемой выражением (8) с $A_{\alpha}$ из формулы (17), где матрицы $U_{\alpha}$ заданы в (50). Обобщенное векторное поле $\omega_{R}$, записанное в виде эволюционного представления, и его расширение принимают вид (6). Тогда условие (8) приводит к следующему ограничению на функцию $R[\theta]$ :

$$
\operatorname{pr} \omega_{R}\left(\theta_{x x}-\frac{1}{2} f^{\prime}(\theta)\right)=D_{x}^{2} R-\frac{1}{2} f^{\prime \prime}(\theta) R=0, \quad \text { если } \quad \theta_{x x}-\frac{1}{2} f^{\prime}(\theta)=0 .
$$

Два частных решения определяющего уравнения (55) имеют следующий вид:

$$
\begin{array}{r}
\omega_{R^{1}}=R^{1}[\theta] \partial_{\theta}, \quad R^{1}[\theta]=\theta_{x} ; \\
\omega_{R^{2}}=R^{2}[\theta] \partial_{\theta}, \quad R^{2}[\theta]=\theta_{x} \int_{\theta_{0}}^{\theta}[f(s)]^{-3 / 2} d s .
\end{array}
$$

Легко проверить, что $\omega_{R^{1}}$ является общей симметрией как для ЛС3 (47), так и для YHK (49):

$$
\begin{aligned}
\operatorname{pr} \omega_{R^{1}}\left(\theta_{x x}-\frac{1}{2} f^{\prime}(\theta)\right) & =0, \quad \text { если } \quad \theta_{x x}-\frac{1}{2} f^{\prime}(\theta)=0, \\
\operatorname{pr} \omega_{R^{1}}\left(\theta_{x}^{2}-f\right) & =0, \quad \text { если } \quad \theta_{x}^{2}-f=0 .
\end{aligned}
$$


Согласно предложению 1 существует двумерная поверхность, которая определяется функцией погружения

$$
F^{R^{1}}([\theta], y, \lambda)=\Phi^{-1} D_{x} \Phi \in \mathfrak{s l}(2, \mathbb{R})
$$

с линейно независимыми векторами

$$
D_{x} F^{R^{1}}=\Phi^{-1}\left(D_{x} U_{1}\right) \Phi, \quad D_{y} F^{R^{1}}=\Phi^{-1}\left(D_{x} U_{2}\right) \Phi .
$$

Во втором случае векторное поле $\omega_{R^{2}}$ является симметрией УНК (49), но не симметрией ЛС3 (47):

$$
\begin{array}{cl}
\operatorname{pr} \omega_{R^{2}}\left(\theta_{x x}-\frac{1}{2} f^{\prime}(\theta)\right) & =0, \quad \text { если } \quad \theta_{x x}-\frac{1}{2} f^{\prime}(\theta)=0, \\
\operatorname{pr} \omega_{R^{2}}\left(\theta_{x}^{2}-f\right) & \neq 0, \quad \text { если } \quad \theta_{x}^{2}-f=0 .
\end{array}
$$

Отсюда следует, что интегральная форма поверхности, связанной с симметрией $\omega_{R^{2}}$, не является формой Фокаса-Гельфанда. Согласно предложению 2 функция погружения двумерной поверхности принимает вид

$$
F^{R^{2}}([\theta], y, \lambda)=\Phi^{-1}\left(\operatorname{pr} \omega_{R^{2}} \Phi\right) \in \mathfrak{s l}(2, \mathbb{R}),
$$

что отвечает линейно независимым касательным векторам

$$
\begin{aligned}
& D_{x} F^{R^{2}}=\Phi^{-1}\left\{\left(\operatorname{pr} \omega_{R^{2}} U_{1}\right)+\operatorname{pr} \omega_{R^{2}}\left(D_{x} \Phi-U_{1} \Phi\right)\right\}, \\
& D_{y} F^{R^{2}}=\Phi^{-1}\left\{\left(\operatorname{pr} \omega_{R^{2}} U_{2}\right)+\operatorname{pr} \omega_{R^{2}}\left(D_{y} \Phi-U_{2} \Phi\right)\right\},
\end{aligned}
$$

где действие $\operatorname{pr} \omega_{R^{2}}$ на ЛС3 (47) (с матричными функциями $U_{\alpha}$, заданными в (50)) не обращается в нуль для всех решений $\Phi$ ЛСЗ:

$$
\begin{aligned}
& \operatorname{pr} \omega_{R^{2}}\left(D_{x} \Phi-U_{1} \Phi\right)=\frac{\theta_{x}}{2(\theta+\lambda)^{3 / 2} f(\theta)^{1 / 2}}\left(\begin{array}{cc}
-\psi_{+}-\psi_{-} & a^{-1}\left(\psi_{+}-\psi_{-}\right) \\
0 & \psi_{+}+\psi_{-}
\end{array}\right), \\
& \operatorname{pr} \omega_{R^{2}}\left(D_{y} \Phi-U_{2} \Phi\right)=\frac{\theta_{x}}{(\theta+\lambda)^{1 / 2} f(\theta)^{1 / 2}}\left(\begin{array}{cc}
-\psi_{+}-\psi_{-} & a^{-1}\left(\psi_{+}-\psi_{-}\right) \\
0 & \psi_{+}+\psi_{-}
\end{array}\right) .
\end{aligned}
$$

В обоих случаях можно найти явные выражения для погружений (57) и (59), но они весьма громоздки, поэтому мы их здесь опускаем.

\section{5. $\mathbb{C} P^{N-1}$-СИГМА-МОДЕЛЬ И СОЛИТОННЫЕ ПОВЕРХНОСТИ}

Рассмотрим $\mathbb{C} P^{N-1}$-модель в терминах эрмитова проектора $P$ ранга 1 :

$$
\begin{gathered}
{\left[\partial_{+} \partial_{-} P, P\right]=\varnothing, \quad \partial_{ \pm}=\frac{1}{2}\left(\partial_{1} \pm i \partial_{2}\right),} \\
P^{2}=P^{\dagger}=P, \quad \operatorname{tr} P=1, \quad \partial_{1}=\partial_{\xi_{1}}, \partial_{2}=\partial_{\xi_{2}} .
\end{gathered}
$$

Мы считаем, что эта модель определена на римановой сфере $S^{2}=\mathbb{C} \cup\{\infty\}$ и что ее функционал действия конечен [10]. Существуют повышающий и понижающий операторы решений системы (62), и любое решение можно записать как повышающий 
оператор, действующий на голоморфное решение [11]:

$$
\begin{array}{r}
\Pi_{ \pm}(P)=\left\{\begin{array}{lr}
\frac{\left(\partial_{ \pm} P\right) P\left(\partial_{\mp} P\right)}{\operatorname{tr}\left(\partial_{ \pm} P P \partial_{\mp} P\right)}, & \left(\partial_{ \pm} P\right) P \neq \varnothing, \\
\varnothing, & \left(\partial_{ \pm} P\right) P=\varnothing,
\end{array}\right. \\
\Pi_{-}^{k+1}(P)=0 \quad \text { для некоторых } k \in \mathbb{Z}^{+} .
\end{array}
$$

ЛС3 записывается как [12]

$$
\partial_{ \pm} \Phi=U_{ \pm} \Phi, \quad U_{ \pm}=\frac{2}{1 \pm \lambda}\left[\partial_{ \pm} P, P\right] \in \mathfrak{s u}(N)
$$

(знак плюс выбирается при $\alpha=1$, минус - при $\alpha=2$ ); ее решение $\Phi=\Phi([P], \lambda)$ переходит в $\mathbb{I}_{N}$ при $\lambda \rightarrow \infty[10],[12]:$

$$
\Phi_{k}=\mathbb{I}_{N}+\frac{4 \lambda}{(1-\lambda)^{2}} \sum_{j=0}^{k-1} \Pi_{-}^{j} P-\frac{2}{1-\lambda} P_{k} \in S U(N), \quad \lambda=i t, \quad t \in \mathbb{R},
$$

где $\mathbb{I}_{N}$ - единичная $(N \times N)$-матрица. Для простоты в этом разделе мы не пишем индекс $k$ у каждого из $N$ проекторов $P_{k}$. Мы выражаем модель в терминах элементов алгебры $\mathfrak{s u}(N)$ вместо $P$ :

$$
\theta \equiv i\left(P-\frac{1}{N} \mathbb{I}_{N}\right) \in \mathfrak{s u}(N)
$$

с алгебраическим ограничением

$$
\theta^{2}=-i \frac{2-N}{N} \theta+\frac{1-N}{N^{2}} \mathbb{I}_{N} \quad \Longleftrightarrow \quad P^{2}=P .
$$

Уравнения Эйлера-Лагранжа принимают вид

$$
\Omega^{j}[\theta]=\left[\left(\partial_{1}^{2}+\partial_{2}^{2}\right) \theta, \theta\right]^{j}=0, \quad j=1, \ldots, N^{2}-1,
$$

где $[\cdot]^{j}-$ коэффициенты $j$-го базисного элемента $e_{j}$ алгебры $\mathfrak{s u}(N)$. Матрицы потенциала $U_{\alpha}$, выраженные в терминах $\theta$, суть

$$
\begin{aligned}
U_{1} & =\frac{-2}{1-\lambda^{2}}\left(\left[\partial_{1} \theta, \theta\right]-i \lambda\left[\partial_{2} \theta, \theta\right]\right) \in \mathfrak{s u}(N), \quad \lambda=i t, \quad t \in \mathbb{R} . \\
U_{2} & =\frac{-2}{1-\lambda^{2}}\left(i \lambda\left[\partial_{1} \theta, \theta\right]+\left[\partial_{2} \theta, \theta\right]\right) \in \mathfrak{s u}(N), \quad
\end{aligned}
$$

Записывая функцию $\Phi$ в терминах $\theta$, мы получаем

$$
\Phi([\theta], \lambda)=\mathbb{I}_{N}+\frac{4 \lambda}{(1-\lambda)^{2}} \sum_{j=0}^{N} \Pi_{-}^{j}\left(\frac{1}{N} \mathbb{I}_{N}-i \theta\right)-\frac{2}{1-\lambda}\left(\frac{1}{N} \mathbb{I}_{N}-i \theta\right) \in S U(N) .
$$

Для любых функций $f$ и $g$ одной переменной уравнения Эйлера-Лагранжа (68) и их ЛС3 (63) (с матрицами потенциала (69)) допускают конформные симметрии

$$
\omega_{C_{i}}=\left[f\left(\xi_{i}\right) \theta_{1}^{j}+g\left(\xi_{i}\right) \theta_{2}^{j}\right] \frac{\partial}{\partial \theta^{j}}, \quad i=1,2 .
$$


Векторные поля $\omega_{C_{i}} \tau$-связаны с полями

$$
\eta_{C_{i}}=\left(\partial_{i} \Phi^{j}\right) \frac{\partial}{\partial \Phi^{j}}+\left(\partial_{i} U_{\alpha}^{j}\right) \frac{\partial}{\partial U_{\alpha}^{j}}, \quad i=1,2,
$$

которые являются конформными симметриями ЛС3 (63). Интегральная форма двумерной поверхности дается с помощью формулы Фокаса-Гельфанда

$$
F=\frac{\Phi^{-1}}{1-\lambda^{2}}\left\{f\left(\xi_{1}\right)\left(\left[\partial_{1} \theta, \theta\right]-i \lambda\left[\partial_{2} \theta, \theta\right]\right)+g\left(\xi_{2}\right)\left(i \lambda\left[\partial_{1} \theta, \theta\right]+\left[\partial_{2} \theta, \theta\right]\right)\right\} \Phi \in \mathfrak{s u}(N) .
$$

\section{6. ЗАКЛЮЧИТЕЛЬНЫЕ ЗАМЕЧАНИЯ}

Подведем итог и сформулируем результаты, полученные в этой работе.

1. Мы применили методику Фокаса-Гельфанда для построения солитонных поверхностей, связанных с обыкновенными дифференциальными уравнениями и дифференциальными уравнениями в частных производных, допускающими представление Лакса.

2. Мы показали, как определить общие симметрии двумерных интегрируемых систем и их ЛСЗ так, чтобы была применима формула Фокаса-Гельфанда для погружения двумерных поверхностей.

3. Мы показали, что существование общих симметрий является необходимым и достаточным условием существования функции погружения в виде, данном формулой Фокаса-Гельфанда (26), с касательными векторами, задаваемыми выражением $(35)$, где векторное поле $\omega_{R}$ является общей симметрией как УНК $\Omega[\theta]=0$, так и ЛСЗ $\Lambda([\theta], \lambda)=0$.

4. Мы установили явное выражение, которое позволяет различать случаи, когда возможна или невозможна интерпретация солитонных поверхностей с помощью формулы (26) с касательными векторами, задаваемыми выражением (38), где векторное поле $\omega_{R}$ является обобщенной симметрией УНК $\Omega[\theta]=0$, но не обязательно симметрией ЛС3 $\Lambda([\theta], \lambda)=0$.

\section{Общие симметрии}

ПРИЛОЖЕНИЕ

1. Пусть $L_{1}$ - максимальная конечномерная подалгебра точечных симметрий Ли, связанная с нелинейными дифференциальными уравнениями $\Omega[\theta]=0$.

2. Пусть $L_{2}$ - максимальная конечномерная подалгебра точечных симметрий Ли линейной системы, связанной с нелинейными дифференциальными уравнениями в частных производных.

3. Пусть $\pi$ является проектором, который действует на совокупность векторных полей $\left\{\omega_{1}, \ldots, \omega_{s}\right\}$ (их оболочка образует множество $L_{2}$ ) так, что

$$
\pi\left(\omega_{k}\right)=\omega_{k} \rho, \quad \rho=x^{i} \partial_{x^{i}}+\theta^{k} \partial_{\theta^{k}}, \quad k=1, \ldots, s,
$$

где $w$ - дифференциальный оператор по всем независимым и зависимым переменным со свойством

$$
\pi^{n}\left(\omega_{k}\right)=\pi\left(\omega_{k}\right), \quad n \in \mathbb{Z}^{+}, \quad k=1, \ldots, s .
$$


4. Общие симметрии нелинейных дифференциальных уравнений в частных производных и ЛСЗ являются векторными полями $\omega_{k}$, оболочка которых образует множество $L_{3}=L_{1} \cap \pi\left(L_{2}\right) \neq \varnothing$. При этом множество $L_{3}$ не обязательно является алгеброй.

Если продолжение одного из векторных полей $\omega_{k}$ из $L_{3}$, действующих на ЛСЗ, должно обратиться в нуль для всех волновых функций $\Phi$, то интегральная форма двумерной поверхности в алгебре Ли дается формулой погружения Фокаса-Гельфанда

$$
F=\Phi^{-1}\left[\beta(\lambda) D_{\lambda} \Phi+S \Phi+\operatorname{pr} \omega_{R} \Phi\right] \in \mathfrak{g},
$$

когда касательные векторы $D_{\alpha} F=\Phi^{-1} A_{\alpha} \Phi$ линейно независимы с матричными функциями

$$
A_{\alpha}=\beta(\lambda) D_{\lambda} U_{\alpha}+\left(D_{\alpha} S+\left[S, U_{\alpha}\right]\right)+\operatorname{pr} \omega_{R} U_{\alpha} \in \mathfrak{g},
$$

где векторное поле $\omega_{R}$ является общей симметрией как УНК $\Omega[\theta]=0$, так и ЛСЗ $\Lambda([\theta], \lambda)=0$.

\section{Список литературы}

[1] A. M. Grundland, S. Post, D. Riglioni, J. Phys. A: Math. Theor., 47:1 (2014), 015201, $14 \mathrm{pp}$.

[2] A. S. Fokas, I. M. Gel'fand, F. Finkel, Q. M. Liu, Selecta Math. (N. S.), 6:4 (2000), 347-375.

[3] A. S. Fokas, I. M. Gelfand, Commun. Math. Phys., 177:1 (1996), 203-220.

[4] A. Sym, Lett. Nuovo Cimento (2), 33:12 (1982), 394-400.

[5] J. Cieśliński, J. Math. Phys., 38:8 (1997), 4255-4272.

[6] A. Doliwa, A. Sym, "Constant mean curvature surfaces in $E^{3}$ as an example of soliton surfaces", Nonlinear Evolution Equations and Dynamical Systems (Baia Verde, Italy, 19-29 June, 1991), eds. M. Boiti, L. Martina, F. Pempinelli, World Sci., Singapore, 1992, 111-117.

[7] П. Олвер, Приложение групп Ли к дифференциальным уравнениям, Мир, М., 1989.

[8] A. M. Grundland, S. Post, J. Phys. A: Math. Theor., 44:16 (2011), 165203, 31 pp.

[9] A. M. Grundland, S. Post, J. Phys. A: Math. Theor., 45:1 (2012), 015204, 20 pp.

[10] A. Din, W. J. Zakrzewski, Nucl. Phys. B, 174:2-3 (1980), 397-406.

[11] P. P. Goldstein, A. M. Grundland, J. Phys. A: Math. Theor., 43:26 (2010), 265206, 18 pp.

[12] В. Е. Захаров, А. В. Михайлов, ЖЭТФ, 74:6 (1978), 1953-1973. 